\title{
UPPER AIRWAY INFECTIONS
}

\section{INTRODUCTION}

As a group, upper respiratory tract infections in children are responsible for approximately 22 million days of school absence and contribute to work loss due to absence of the family/caregiver caring for ill children. Children under six years of age average six to eight upper respiratory tract infections per year. Although these infections are usually selflimited, they can be associated with airway obstruction and may be life-threatening. Laryngotracheobronchitis (croup) is a common cause of upper airway obstruction in children, affecting up to $6 \%$ of children under six years of age. Although less than 5\% of children with croup are hospitalized, croup account for 35,000 hospital admissions annually and results in the need for endotracheal intubation for 1-2\% of those hospitalized. Other upper airway infections that may lead to airway obstruction include epiglottitis, bacterial tracheitis, severe tonsillitis, and deep neck abscesses. Pediatric hospitalists commonly encounter these patients and are often in the best position to coordinate care across multiple specialties when necessary. Pediatric hospitalists should be able to recognize signs and symptoms of impending or actual airway obstruction, provide immediate care, and arrange for the appropriate subsequent level of care.

\section{KNOWLEDGE}

Pediatric hospitalists should be able to:

- Describe the anatomy of the upper respiratory tract and discuss how abnormalities in airflow in different locations may alter clinical presentation.

- Compare and contrast the airway anatomy of neonates, infants, toddlers, preschoolers, school aged children, and adolescents.

- Differentiate between the common infectious etiologies of upper airway obstruction in children of various ages.

- Review alternate diagnoses which may mimic the presentation of acute upper respiratory infection such as allergic reaction, toxic inhalant exposure, and others.

- Describe the signs and symptoms of upper airway obstruction, such as stertor, stridor, "tripod positioning," dysphagia, drooling, trismus and others.

- List the types of radiographic studies available to assess the upper airway (such as plain radiographs, ultrasonography, computed tomography, and magnetic resonance imaging) and discuss the risks, benefits, and indications for each.

- Discuss the indications for nebulized epinephrine, glucocorticoids, antibiotics, and other medications in the treatment of upper respiratory tract infections.

- Compare and contrast the benefits and limitations of various modalities of airway stabilization and respiratory support in patients with varying degrees of upper airway obstruction.

- List the indications for hospital admission, and explain the utility of various monitoring options.

- Discuss the changes in clinical status that indicate need for escalation of care, such as worsening stridor or work of breathing, decreased air entry, cyanosis, altered mental status and others.

- Describe the criteria for management in an intensive care unit or transfer to a tertiary care facility.

- Review the indications for emergent surgical consultation.

- List the criteria for hospital discharge, attending to change in symptoms, oxygenation, hydration, and education.

\section{SKILLS}

Pediatric hospitalists should be able to:

- Perform an appropriately focused medical history, attending to symptoms of potential airway obstruction.

- Conduct an appropriate physical examination in children with upper respiratory tract infection, attending to signs and symptoms that may indicate the etiology or severity of the infection.

- Consistently adhere to infection control practices.

- Correctly identify patients with co-morbidities or potential underlying anatomic abnormalities and order appropriate testing and treatment.

- Identify complications of the infection and respond with appropriate actions.

- Perform an evidence-based, cost-effective diagnostic evaluation and treatment plan, avoiding unnecessary testing as appropriate.

- Order appropriate monitoring and correctly interpret monitor data. 
- Perform careful reassessments daily and as needed, note changes in clinical status and respond with appropriate actions and escalation of care as appropriate.

- Stabilize the airway and provide appropriate respiratory support for patients with impending or actual airway obstruction or respiratory failure, or arrange for the appropriate personnel to perform the procedure in a timely and safe manner.

- Recognize the indications for and efficiently obtain subspecialty consultation.

\section{ATTITUDES}

Pediatric hospitalists should be able to:

- Role model and advocate for strict adherence to infection control practices.

- Communicate effectively with patients and the family/caregiver regarding the diagnosis, management plan, and follow-up needs.

- Collaborate with the primary care provider and subspecialists to ensure a coordinated discharge.

\section{SYSTEMS ORGANIZATION AND IMPROVEMENT}

In order to improve efficiency and quality within their organizations, pediatric hospitalists should:

- Lead, coordinate or participate in the development and implementation of cost-effective, safe, evidence-based care within a multidisciplinary team for hospitalized children with upper respiratory tract infections.

- Collaborate with hospital administration and community partners to develop and sustain referral networks between local facilities and tertiary referral centers for hospitalized patients with upper respiratory tract infections. 